\title{
Prediction of fertility by mating latency and photoperiod in nulliparous and primiparous meadow voles (Microtus pennsylvanicus)
}

\author{
L. R. Meek and T. M. Lee* \\ Department of Psychology Laboratory Building, 1103 E. Huron, Neuroscience University of \\ Michigan, Ann Arbor, MI 48104-1687, USA
}

\begin{abstract}
Mating behaviour and litter production of female meadow voles (Microtus pennsylvanicus) housed in long ( $14 \mathrm{~h}$ light:10 h dark; long day; LD) or short ( $10 \mathrm{~h}$ light:14 h dark; short day; SD) photoperiods were monitored to determine whether the reduced birthrate of SD females resulted from a lack of copulation. All females mated, but fewer SD females gave birth. LD and SD females fell into three distinct groups based on mating latency. The rapid onset group (RO) mated between $7 \mathrm{~min}$ and $9 \mathrm{~h}$ after pairing, the intermediate onset group (IO) mated between 16-44 h and the late onset group (LO) mated after 58-262 h of male contact. Sixtyseven per cent of LD females were assigned to group RO, $27 \%$ to IO, and $6 \%$ to LO. In contrast, $30 \%$ of SD females were assigned to group RO, $35 \%$ to IO and $35 \%$ to LO. Fertility was predicted by mating latency. Sixty-nine per cent of RO, 93\% of IO and $33 \%$ of LO animals gave birth. In a further experiment, a small-mouthed cup was added to the environment to serve as an escape for females wishing to avoid mating. Although females did not use the cup to escape male approaches, mating occurred in only $66 \%$ of SD females, but was observed in all LD females. In a final experiment, mating latency and litter production were recorded in primiparous $L D$ and $S D$ females initially observed in the first experiment. Group LO was eliminated in parous females; all primiparous LD and SD females mated within $48 \mathrm{~h}$. Birthrates of LD $(82 \%)$ and SD $(73 \%)$ parous females were increased compared with birthrates of nulliparous females ( $\mathrm{LD}=65 \%$; $\mathrm{SD}=55 \%$ ). These observations suggest that long daylength and parity increase spontaneous oestrus in meadow voles $(50 \%$ of nulliparous and $80 \%$ of primiparous $\mathrm{RO}$ animals mated in less than $1.5 \mathrm{~h}$ ). Females in the IO and LO groups are probably induced into oestrus, as normally described for arvicoline rodents, by direct male contact. Rapid mating $(<48 \mathrm{~h})$ predicts greater fertility for both LD and SD females, while delayed mating $(>58 \mathrm{~h})$ predicts low fertility. Parity decreases mating latency and increases litter production. Short day females produce fewer litters than LD females in equivalent groups.
\end{abstract}

\section{Introduction}

Female voles (Microtus) are thought to be induced into behavioural oestrus by exposure to the urine of reproductively competent males (Sawrey and Dewsbury, 1985). Baddaloo and Clulow (1980) demonstrated that young female meadow voles (Microtus pennsylvanicus) develop heavier uteri and ovaries after exposure to a mature male, suggesting that behavioural oestrus in meadow voles also depends upon male chemical stimulation. As in many other species of vole, the induction of ovulation by copulatory stimulation is well documented in meadow voles (Lee and Horvath, 1969; Clulow and Mallory, 1970; Lee et al., 1970). This mode of induction of oestrus and ovulation suggests that most mature healthy female meadow voles exposed to a reproductively competent male should become pregnant.

${ }^{*}$ Correspondence.

Received 11 March 1992
However, litter production in laboratory-housed females kept under short winter-like photoperiod conditions $(10 \mathrm{~h} \mathrm{light:}$ $14 \mathrm{~h}$ dark; short day; SD) paired with reproductively competent males that have been housed in long summer-like photoperiods ( $14 \mathrm{~h}$ light: $10 \mathrm{~h}$ dark; long day; $\mathrm{LD}$ ) is low; only $20-30 \%$ of SD females give birth (Imel and Amann, 1979; Lee et al., 1987). Field studies indicate that winter breeding occurs; $0-50 \%$ of females are found pregnant in the winter of any given year (Christian, 1971, 1980). In contrast, more than $95 \%$ of females in the field become pregnant during the summer (McShea and Madison, 1989) and 65-90\% of laboratory females housed in LD give birth (Imel and Amann, 1979; Lee et al., 1987). This suggests that adult female meadow voles do not rely solely on male photoperiodic responses (regression of testes in response to short days) to limit litter production, but possess an independent photoperiod-sensitive mechanism enabling them to avoid pregnancy under predictable, adverse conditions such as winter.

Short-day females may avoid pregnancy through a variety of behavioural or physiological methods. The most straight- 
forward mechanism would be insensitivity to oestrus-inducing male urine, resulting in fewer matings. Alternatively, females may be induced into oestrus by males and mate, but limit fertility through post-copulatory mechanisms.

The experiments reported here were designed to investigate whether SD females avoid pregnancies through a reduction or alteration in mating behaviour. Experiment 1 investigated the mating behaviour and subsequent fertility of mature, nulliparous female meadow voles housed under long or short daylength conditions. Experiment 2 investigated whether increased environmental complexity, which allowed females to avoid copulation, resulted in reduced fertility in SD females compared with $L D$ females. It is well known that parity increases fertility in rodents (Bronson, 1989), and previous work in both voles and non-microtines indicates that photoperiod has no impact on postpartum oestrus (Tamarin, 1977; Charlton et al., 1978; Beasley et al., 1981). Meadow voles, however, may not have access to fertile males during the winter at the time of a postpartum oestrus. In Expt 3 we therefore examined the influence of photoperiod on mating behaviour and fertility in parous females, 8 weeks after their first litter was removed.

\section{Materials and Methods}

\section{Animals}

At weaning ( 3 weeks of age), 96 virgin female voles (Microtus pennsylvanicus) born and raised under long day conditions ( $14 \mathrm{~h}$ light:10 $\mathrm{h}$ dark; LD) from the laboratory colony at the University of Michigan either remained under long day conditions $(n=53)$ or were placed in short photoperiod conditions (10 h light:14 h dark; short day, SD; $n=43$ ) for 8 weeks. Females were near to sexual maturity at 3 weeks of age, as determined by perforate vaginae. Animals were housed individually or in same sex pairs with Purina Mouse Chow (no. 5015; Purina Mills, Inc., St Louis, $\mathrm{MO}$ ) and water was available ab libitum.

\section{Videotaping procedure}

After exposure to the experimental photoperiod for 8 weeks, each female was placed in a 10 gallon aquarium with an LD male known to have sired litters with both LD and SD females. All females had perforate vaginae at this time. Pairs were maintained in the experimental photoperiod of the fermale. Sawdust bedding was provided and was not changed while animals were paired. The outside of the aquarium was masked with paper to minimize disturbance.

The behaviour of each pair was videotaped continuously with a Panasonic Low Light Camera and extended-play VCR. A $25 \mathrm{~W}$ red light provided illumination for the camera during the dark portion of the photoperiod.

Pairs were videotaped until post-copulation satiety occurred or for 2 weeks, whichever occurred first. All pairs in Expt I were videotaped for $12-24 \mathrm{~h}$ after copulation had ceased to allow for the possibility that copulatory activity would resume after a lengthy hiatus. No pairs were observed to resume mating after a $4 \mathrm{~h}$ hiatus; thus for Expts 2 and 3, satiety was defined as $4 \mathrm{~h}$ without mating. Within $24 \mathrm{~h}$ of copulation, females were separated from the male and remained in the same photoperiod for 3 weeks or until litters were produced.

\section{Mating behaviour}

For each pair, mating latency, frequency of mounts, intromissions and ejaculations, the total length of all ejaculatory series (including and excluding the post-ejaculatory interval), mean inter-intromission interval and litter production were recorded. Mating latency and litter production only are described in this paper. Mating latency was defined as the time from initial pairing until the first intromission. Litter production was defined as production of pups 3 weeks after copulation.

\section{Statistical analysis}

Mating latency was analysed using a two-tailed Student's $t$ test. Bartlett's chi square was used to measure homogeneity of variance. Differences in litter production were analysed by chisquare analyses. Probability values less than or equal to 0.05 were considered significant. Values are given as means \pm SEM.

\section{Experiment 1}

LD $(n=30)$ and SD $(n=20)$ animals were paired with LD males and videotaped as stated in the videotaping procedure.

\section{Experiment 2}

$\mathrm{LD}(n=12)$ and SD $(n=12)$ females were paired with $\mathrm{LD}$ males and videotaped as in Expt 1. A small-mouthed container $(4.4 \mathrm{~cm}$ diameter at the mouth, $10 \mathrm{~cm}$ in length) open at one end and large enough for only one vole to enter was added to the environment. Mating latency and litter production was monitored as in Expt 1.

\section{Experiment 3}

After pups were weaned, 22 LD primiparous females from Expt 1 were kept under LD $(n=11)$ or $\mathrm{SD}(n=11)$ conditions for 8 weeks. They were then paired with a male, videotaped and maintained in the photoperiod of mating until pups were weaned as previously described.

\section{Results}

\section{Experiment 1}

All animals mated within 2 weeks. However, mating latency varied greatly between $\mathrm{LD}$ and $\mathrm{SD}$ animals. SD females began mating significantly later than LD animals (LD $=805.1 \pm$ $289.5 \mathrm{~min} ; \mathrm{SD}=3886.4 \pm 1131.1 \mathrm{~min}, P<0.05)$. This difference was significant despite the lack of homogeneity of variance $(P<0.05)$. All LD females mated within 3.2 days; $50 \%$ mated in less than $1.5 \mathrm{~h}$ (range $=7-4648 \mathrm{~min}$ ). In contrast, only five 


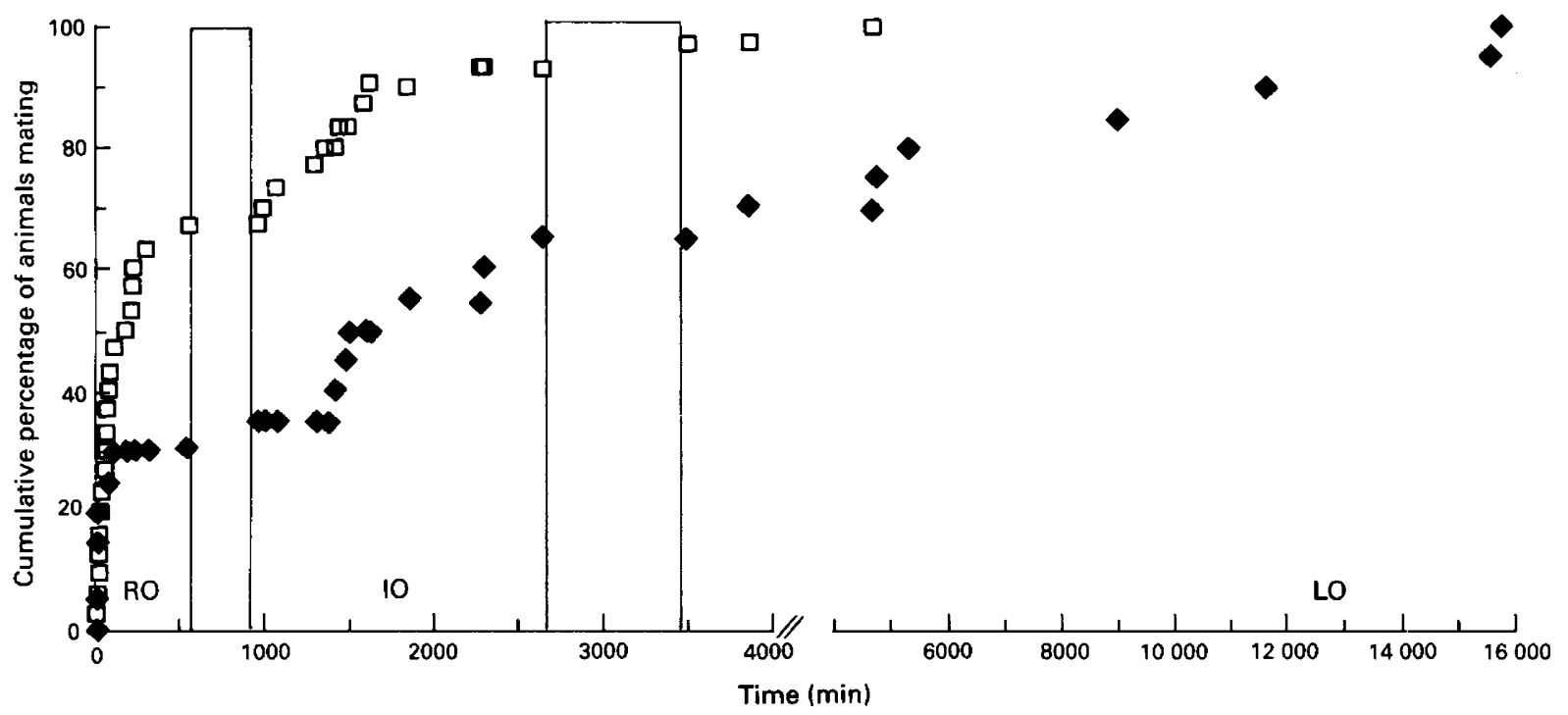

Fig. 1. Cumulative increase in the percentage of $(\square)$ LD (long day; $14 \mathrm{~h}$ light:10 h dark) and ( $\bullet$ SD (short day; $10 \mathrm{~h}$ light:14 h dark) female meadow voles mating in Expt 1 with time. Columns denoting periods of no mating by LD and SD females separate groups RO, IO and LO. The first column corresponds to $7 \mathrm{~h}$, the second to $14.4 \mathrm{~h}$. RO: rapid onset group; IO: intermediate onset group; LO: late onset group.

of $20 \mathrm{SD}$ females mated within $1.5 \mathrm{~h}$ (range $=8-15720 \mathrm{~min}$ ). Ranking animals by mating latency produced three distinct mating patterns (Fig. 1).

The rapid onset group (RO) comprised 26 animals $(77 \% \mathrm{LD}$; $23 \% \mathrm{SD}$ ) mating within $9 \mathrm{~h}$ after pairing with a male. The intermediate onset group (IO) contained 15 animals (53\% LD; $47 \%$ $\mathrm{SD}$ ) mating between 16 and $44 \mathrm{~h}$ after pairing, whereas the late onset group (LO) consisted of nine animals ( $22 \% \mathrm{LD} ; 78 \% \mathrm{SD}$ ) mating 58-262 $\mathrm{h}$ after pairing with the male. The divisions between these groups were very clear, with no animals mating between 9 and $16 \mathrm{~h}$, or between 44 and $58 \mathrm{~h}$ after exposure to the male (Fig. I). No other interval of $7 \mathrm{~h}$ without mating occurred before $64 \mathrm{~h}$ after pairing.

Birthrates of $1 O$ females (93\%) were significantly higher than those of LO females $(33 \% ; P<0.05)$ but did not differ from RO females $(69 \%)$. RO and LO birthrates were not significantly different.

When the interaction of photoperiod and mating latency on birthrate was considered, SD birthrates accounted for the low overall birthrate in RO females; $75 \%$ of long day RO females gave birth but only $50 \%$ of short day females produced a litter. Birthrates for $\mathrm{IO}$ females in both photoperiods ( $\mathrm{LD}=100 \%$; $\mathrm{SD}=85 \%$ ) were significantly higher than for LO females ( $\mathrm{LD}=50 \% ; \mathrm{SD}=29 \% ; P<0.05)$. $\mathrm{LD}$ and $\mathrm{SD}$ birthrates did not differ significantly within any mating latency group, although SD birthrates were always lower than LD (Fig. 2).

\section{Experiment 2}

Mating patterns were altered in SD animals when a small container was added to the environment. All LD animals mated but four of 12 SD animals did not mate within 2 weeks. Mating latency was significantly shorter in $\mathrm{LD}$ than in $\mathrm{SD}$ animals $(\mathrm{LD}=$ $530.33 \pm 289.61 \mathrm{~min} ; \mathrm{SD}=2454.13 \pm 968.38 \mathrm{~min}, P<0.05$, excluding the four SD animals that did not mate). When animals

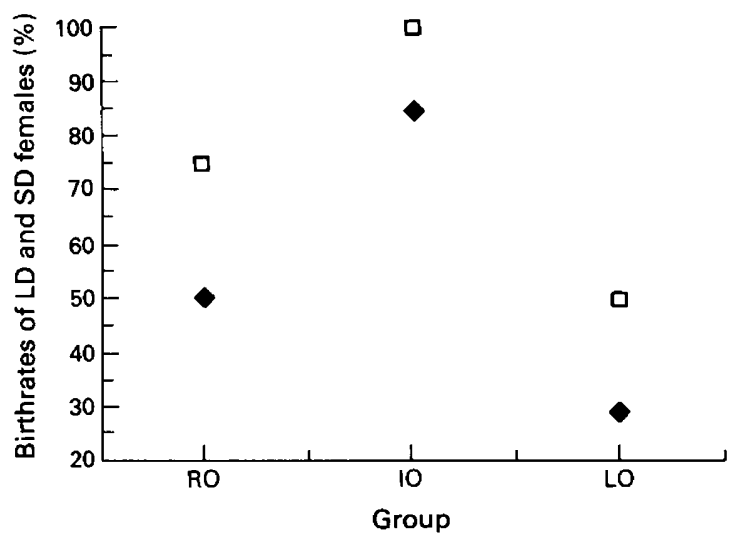

Fig. 2. Comparison of birthrates of ( $\square$ ) LD and ( $\bullet$ ) SD meadow voles in groups RO, IO and LO in Expt 1. RO: rapid onset group; IO: intermediate onset group; LO: late onset group.

were ranked by mating latency, they fell into the same three mating groups as in Expt 1 . The percentage of LD females in each group was similar to Expt $1 ; \mathrm{RO}=67 \% ; \mathrm{IO}=25 \%$; $\mathrm{LO}=8 \%$. However, the percentage of SD females in each mating latency group in Expt 2 differed from that found in Expt 1. Only one of $12 \mathrm{SD}$ females ( $8 \%$ ) was assigned to group RO. In contrast, six of $12(50 \%)$ were in IO and five of $12(42 \%)$ in LO. Owing to the small number of animals involved, the shift in mating latency did not reach statistical significance. Birthrates for SD females in RO, IO and LO were $100 \%, 67 \%$ and $20 \%$, respectively. Short day females that mated (eight of 12) achieved an overall birthrate of $75 \%$. Long day birthrates did not differ from Expt 1 .

\section{Experiment 3}

Mating latency in $L D$ primiparous animals $(192.9 \pm 80.1 \mathrm{~min})$ was shorter than in LD nulliparous females $(805.1 \pm 289.5)$, 


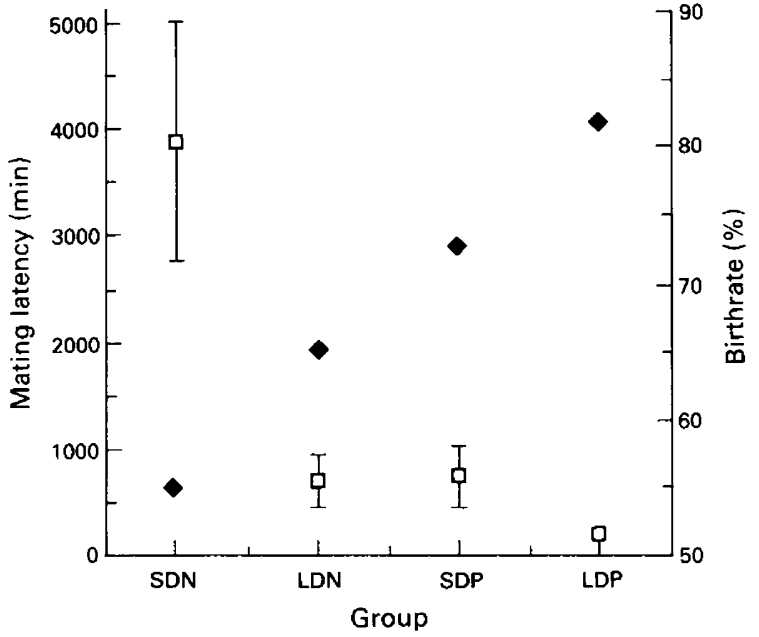

Fig. 3. Comparison of mating latency and percentage of meadow vole females giving birth in Expts 1 and 3. SDN: short day nulliparous fernales; LDN: long day nulliparous females: SDP: short day primiparous females; LDP: long day primiparous females. Increases in birthrates with parity did not reach significance. Mating latency in SDN is significantly longer than all other groups $(P<0.05)$. ( $\square)$ Mating latency; (४) birthrate.

although this difference was not significant $(t=1.399, P=$ 0.196; two-tailed test; Fig. 3). Mean mating latency in SD primiparous females was similar to LD nulliparous animals and significantly shorter than SD nulliparous females $(P<0.05$; Fig. 3). Birthrates in $L D$ and $S D$ primiparous groups were increased compared with nulliparous animals but with only 11 animals per group the differences were not significant (Fig. 3).

When primiparous animals were ranked by mating latency, all animals fell into groups $\mathrm{RO}$ and IO regardless of photoperiod. All LD and SD primiparous animals mated within $48 \mathrm{~h}$; only five animals mated in group IO. Birthrates for primiparous $\mathrm{RO}(82 \%)$ and $\mathrm{IO}(60 \%)$ animals did not differ significantly from each other, or from $\mathrm{RO}$ and $\mathrm{IO}$ birthrates for nulliparous females.

When all animals from the three experiments $(n=96)$ were ranked by mating latency, the divisions into $\mathrm{RO}, \mathrm{IO}$ and LO observed in Expt 1 were preserved, although non-mating intervals were slightly shorter. $\mathrm{RO}$ animals mated between $7 \mathrm{~min}$ and $9.4 \mathrm{~h}$, IO mated between 14.4 and $48 \mathrm{~h}$, and LO mated between $58 \mathrm{~h}$ and 2 weeks.

\section{Discussion}

These results indicate that female meadow voles paired with reproductively competent males mate within three, distinct, non-overlapping periods as defined by mating latency. Furthermore, fertility differs between these three mating latency groups, suggesting that mating latency indicates differences in the hormonal (oestrous) state among the three groups at the time of pairing with the male and predicts subsequent fertility. Short day lengths lengthen mating latency and inhibit fertility; $\mathrm{SD}$ females exhibited longer mating latencies and decreased fertility within each mating latency group than LD females in all three experiments. When mating occurred in a slightly more complex environment, mating latency increased for SD females (four animals never mated), but was unchanged in LD females. For those SD females mating in the complex environment, fertility was increased, although overall fertility was unchanged. In contrast to nulliparous animals, all parous females mated within $48 \mathrm{~h}$ and fertility was greater than that of nulliparous females, suggesting that parity may permanently decrease the responsiveness of female meadow voles to inhibitory short daylengths. Finally, the rapid onset to mating seen in $\mathrm{RO}$ animals $(7 \mathrm{~min}-9.4 \mathrm{~h}$ ) in LD and SD nulliparous females suggests that a large proportion of meadow voles ( $75 \%$ of $L D$ and $30 \%$ of SD) may not need exposure to a male to achieve behavioural oestrus, but exhibit spontaneous oestrus. Parity increased the proportion of females exhibiting spontaneous oestrus to $91 \%$ for $\mathrm{LD}$ and $64 \%$ for SD.

The boundaries of the three mating latency groups, $\mathrm{RO}, \mathrm{IO}$ and LO, were preserved throughout three different paradigms $(n=96)$, suggesting that these differences depend upon hormonal differences among these groups that result in different mating latencies (oestrus) and a different birthrate. Mating latency and birthrate differed markedly in LO animals (primarily SD females), compared with RO or IO animals. $L O$ females are apparently induced into behavioural oestrus (as defined by male approaches and subsequent copulation). However, their long mating latencies and aberrant mating behaviours (L. Meek and T. Lee, unpublished) indicate an abnormal oestrous state with either lower than normal blood concentrations of oestrogen or an insensitivity to the oestrogen. Experiment 2 suggests that, in the field, many of these females would avoid mating. We suggest that the low birthrate of LO females is a direct result of their aberrant mating behaviour (L. Meek and T. Lee, unpublished); that is, the aberrant mating pattern fails to induce the LH surge that is necessary for ovulation.

$\mathrm{RO}$ and IO females successfully produce young in SD or LD photoperiods. These animals may represent two distinct subpopulations, one which needs contact with male chemosignals to achieve oestrus (IO) and one which does not (RO); or they may represent a single group of animals that show a continuum of follicular development. This experiment did not distinguish between these possibilities.

However, the probability of nulliparous females falling into $\mathrm{RO}$ or IO groups was significantly influenced by photoperiod. Most LD females $(67 \%)$ mated in RO with high success rate of litter production $(75 \%)$. Those LD females mating in IO $(27 \%)$ had a statistically equivalent birthrate (100\%). In contrast, only $33 \%$ of SD females in Expt 1 (birthrate $=50 \%$ ) and $8 \%$ in Expt 2 (one animal; a litter was produced) mated in group RO. Those SD females mating in IO had birthrates of $85 \%$ and $67 \%$ (Expts 1 and 2), respectively. These data indicate that SD females are more successful at producing litters if mating is delayed until $14-48 \mathrm{~h}$ after pairing with a male.

If $\mathrm{RO}$ animals have spontaneous oestrus, then long days (Expts 1 and 2) and parity (Expt 3) increase the probability that a female will have spontaneous oestrus and successfully produce a litter. Thus short daylengths probably inhibit spontaneous oestrus in nulliparous females and dramatically increase the number of females that do not mate until after $58 \mathrm{~h}$ of male contact or do not mate at all. Short day females mating in IO have a fertility equivalent to LD animals, whereas fertility is 
very low for those that have not copulated by $48 \mathrm{~h}$ after pairing with a male (LO) or those that copulate before $9 \mathrm{~h}$ of male contact (RO).

Since male approaches to the female and subsequent copulation occur, all SD females are assumed to produce chemosensory or other oestrogen-dependent cues indicating oestrus to the male. The hormonal milieu of RO and IO animals from each photoperiod is probably very similar before and during mating, as their mating sequences are indistinguishable (L. Meek and T. Lee, unpublished). However, the fertility of SD females was subsequently reduced, compared with LD females, indicating that a post-copulatory mechanism may be involved in preventing winter pregnancies. For example, there may be failure of the post-copulatory prolactin rise necessary to maintain corpora lutea (Milligan, 1975; Milligan and MacKinnon, 1976).

The behavioural data indicate that LD and SD nulliparous and primiparous females are uniquely adapted hormonally to winter or summer conditions. Long day female meadow voles mate quickly $(50 \%$ within $1.5 \mathrm{~h})$ and have a high percentage of successful pregnancies in the laboratory. All primiparous females also mate quickly (within $48 \mathrm{~h}$ ) and show increased litter success compared with nulliparous females, suggesting that primiparous female meadow voles do not respond to the inhibitory influence of short days. This lack of response to photoperiod by primiparous females has been noted previously in field voles (Charlton et al., 1978), beach voles (Tamarin, 1977) and white-footed mice (Beasley et al., 1981). Species with short natural lifespans may continue to reproduce despite decreasing daylengths to maximize lifetime litter production.

Under laboratory conditions mimicking the short daylengths of winter, a season when field pregnancies decline significantly, SD nulliparous females show decreased litter production compared with nulliparous LD females in all three mating latency groups. Despite the availability of high-quality food and warm ambient temperatures, short photoperiods delay mating and reduce litter production in nulliparous meadow voles in the laboratory. It appears that most SD females mate if a reproductively competent male is available. However, both in the field and the laboratory, an assessment of parity, photoperiod, temperature, general health, body weight and fat, and available nutrients probably ultimately determines the success of a pregnancy in winter photoperiod.
We would like to thank J. Bacon for her help with these projects, and J. Donner for animal care. This research was supported by an NSF graduate fellowship to L. Meek and an NIH grant (HD-24575) to T. Lee.

\section{References}

Baddaloo EGY and Clulow FV (1980) Effects of the male on growth, sexual maturation, and the ovulation of young female meadow voles, Microtus pennsylvanicus Canadian journal of Zoology 59 415-421

Beasley LJ, Johnston PG and Zucker I (1981) Photoperiodic regulation of reproduction in postpartum Peromyscus leucopus Biology of Reproduction $\mathbf{2 4}$ 962-966

Bronson FH (1989) Mammalian Reproductive Biology The University of Chicago Press, Chicago

Charlton HM, Milligan SR and Versi E (1978) Studies on the control of the corpus luteum in the vole, Microtus agrestis Journal of Reproduction and Fertility 52 283-288

Christian JJ (1971) Fighting, maturity and population density in Microtus pennsylvanicus Joumal of Mammalogy 52 556-567

Christian JJ (1980) Regulation of annual rhythms of reproduction in temperate small rodents. In Testicular Development. Structure and Function pp 367-380 Eds A Steinberger and F Steinberger. Raven Press, NY

Clulow FV and Mallory FF (1970) Oestrus and induced ovulation in the meadow vole, Microtus pennsylvanicus Joumal of Reproduction and Fertility 23 341-343

Imel KJ and Amann RP (1979) Effects of duration of daily illumination on reproductive organs and fertility of the meadow vole Laboratory Animal Srience 29 $182-185$

Lee $\mathrm{C}$ and Horvarth DJ (1969) Management of the meadow vole (Microtus pennsylvanicus) Laboratory Animal Science 19 88-91

Lee C, Horvath DJ, Metcalfe RW and Inskeep EK (1970) Ovulation in Microtus pennsylvanicus in a laboratory environment Laboratory Animal Care 20 1098-1102

Lee TM, Smale L, Zucker I and Dark J (1987) Role of photoperiod during preg. nancy and lactation in the meadow vole (Microtus pennsylvanicus) Journal of Reproduction and Fertility $\mathbf{8 1} 343-350$

McShea WJ and Madison DM (1989) Measurements of reproductive traits in a field population of meadow voles Joumal of Mammalogy 70 132-141

Milligan SR (1975) Mating, ovulation and corpus luteum function in the vole, Microtus agrestis Journal of Reproduction and Fertility 42 35-44

Milligan SR and MacKinnon PCB (1976) Correlation of plasma LH and prolactin levels with the fate of the corpus luteum in the vole, Microtus agrestis Journal of Reproduction and Fertility 47 111-113

Sawrey DK and Dewsbury DA (1985) Control of ovulation, vaginal oestrus and behavioural receptivity in voles (Microtus) Neuroscience and Biobehavioral Review 9 563-571

Tamarin RH (1977) Demography of the beach vole (Microtus breweri) and the meadow vole (Microtus pennsylvanicus) in southeastern Massachusetts Ecology 58 1310-1321 